\title{
Simulation and Optimization in Materials Technology
}

\author{
Martha Guerrero, ${ }^{1}$ Caner Simsir, ${ }^{2}$ Augusto Deus, ${ }^{3}$ and Bozidar Sarler ${ }^{4}$ \\ ${ }^{1}$ Universidad Autonoma de Nuevo Leon, Facultad de Ingenieria Mecanica y Electrica, Avenida Universidad S/N, \\ Cd. Universitaria, CP 66451, San Nicolas de los Garza, NL, Mexico \\ ${ }^{2}$ Atilim University, Metal Forming Center of Excellence (MFCE), Atilim University Campus, Kizilcasar Mahallesi, \\ 06836 Incek-Golbasi, Ankara, Turkey \\ ${ }^{3}$ Universidade de Lisboa, Instituto Superior Tecnico, Departmento de Engenharia Mecanica e ICEMS, Avenida Rovisco Pais, \\ 1049-001 Lisboa, Portugal \\ ${ }^{4}$ University of Nova Gorica, Laboratory for Multiphase Processes, Vipavska 13, 5000 Nova Gorica, \\ Slovenia \& Institute of Metals and Technology, Laboratory for Simulation of Materials and Processes, Lepi pot 11, \\ 1000 Ljubljana, Slovenia
}

Correspondence should be addressed to Martha Guerrero; martha.guerreromt@uanl.edu.mx

Received 11 February 2014; Accepted 11 February 2014; Published 12 March 2014

Copyright (C) 2014 Martha Guerrero et al. This is an open access article distributed under the Creative Commons Attribution License, which permits unrestricted use, distribution, and reproduction in any medium, provided the original work is properly cited.

The interdisciplinary field of materials technology is crucial for development of new materials, facing a huge spectrum of challenges in the area of natural resources, energy, new functionality, environmental impact, and so forth.

Computational modelling and simulation on quantum mechanics, molecular dynamics, and continuum mechanics levels play an ever increasing role in materials technology. Together with automatic computer optimization of different materials technology aspects, they form a framework for virtual design of new materials and processes. This framework is built on multiscale, multiphysics, and multiobjective components. New numerical methods and parallelization on large computers give this research field an additional acceleration. The virtual design avoids many expensive experiments and prototyping. In addition, completely new materials and production processes can be tested well before actual materials synthesis or production process construction.

The present special issue is dedicated to the contemporary efforts in the described field.

Chen Gang at al. present molecular dynamics simulation of solidification, Ali Akbar Lotfi Neyestanak et al. present finite element method analysis of fatigue durability, Anish Kumar et al. present experiments and statistical model of an electrothermal production process, Andrey Gumenyuk and Michael Retheimer present a numerical model of hot cracking, Xueming Hua and Yixiong $\mathrm{Wu}$ present a model for arc interference in welding process, Kan Ankang and Han Houde investigate the effective thermal conductivity of polyurethane foams, Nana Wang and Jinxiang Liu investigate the effect of process parameters of nitriding of cast iron, Yung-Tsan Jou et al. computationally optimize the assembly technology for core circuit board parts, Leandro Neckel et al. model the ballistic impact of a ceramic-metal protection system, Dolly Santos-Barbosa et al. model the influence of process parameters on dimensions of porcelain tiles, E. Güler and M. Güler explore the mechanical properties of Au by using geometry optimization approach, C. Mahesh et al. model thermal conductivity of composite materials, Mile B. Djurdjević et al. use the method of equivalency to describe the liquidus temperature of aluminium and magnesium alloys, Jun Fang et al. model the bending of the steel tubes, and Giuseppe Casalino et al. use neurofuzzy model to predict defects in titanium alloys.

The selected 15 papers for the present special issue demonstrate the power of simulation and optimization in diverse aspects of materials technology.

Martha Guerrero Caner Simsir Augusto Deus Bozidar Sarler 

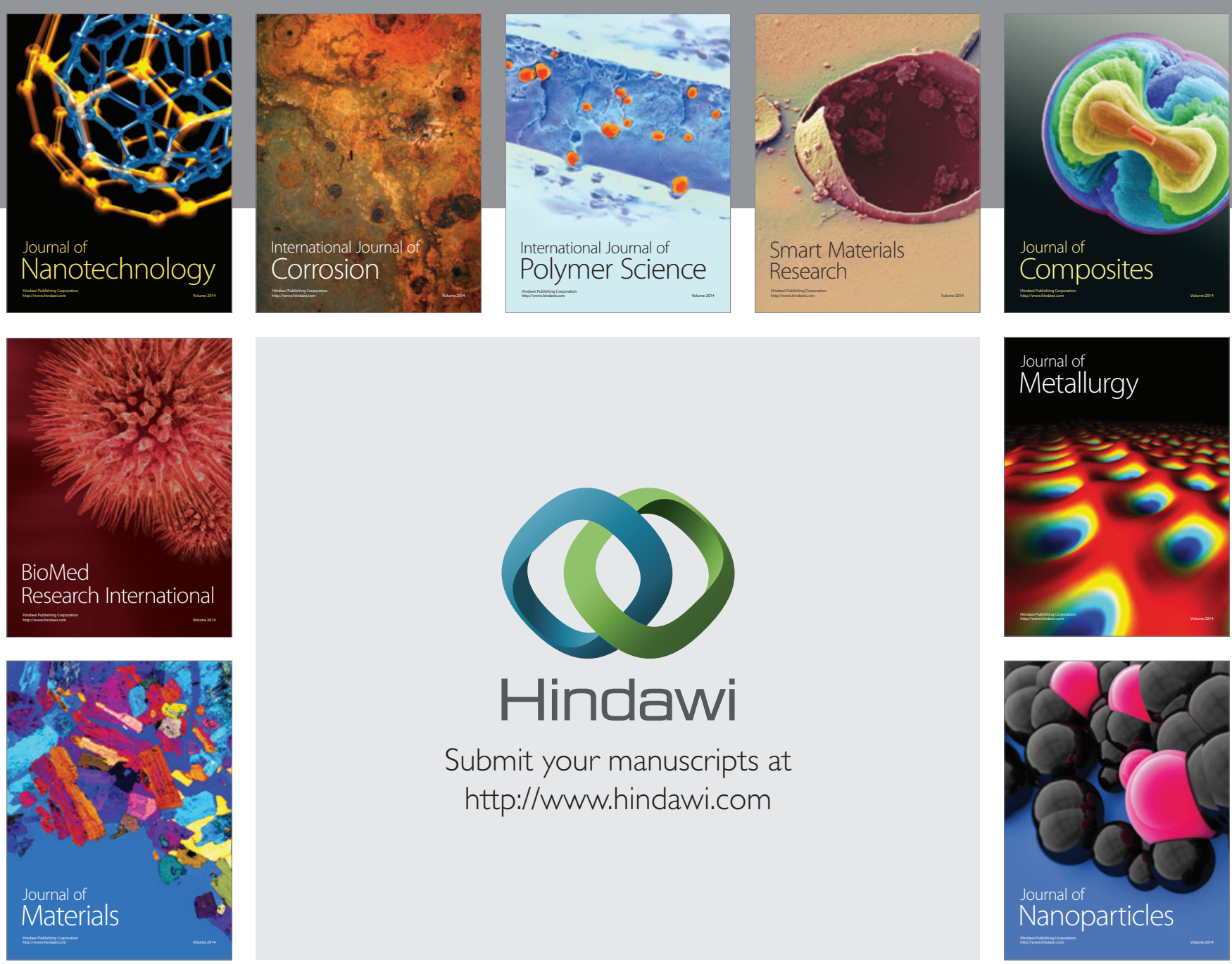

Submit your manuscripts at http://www.hindawi.com
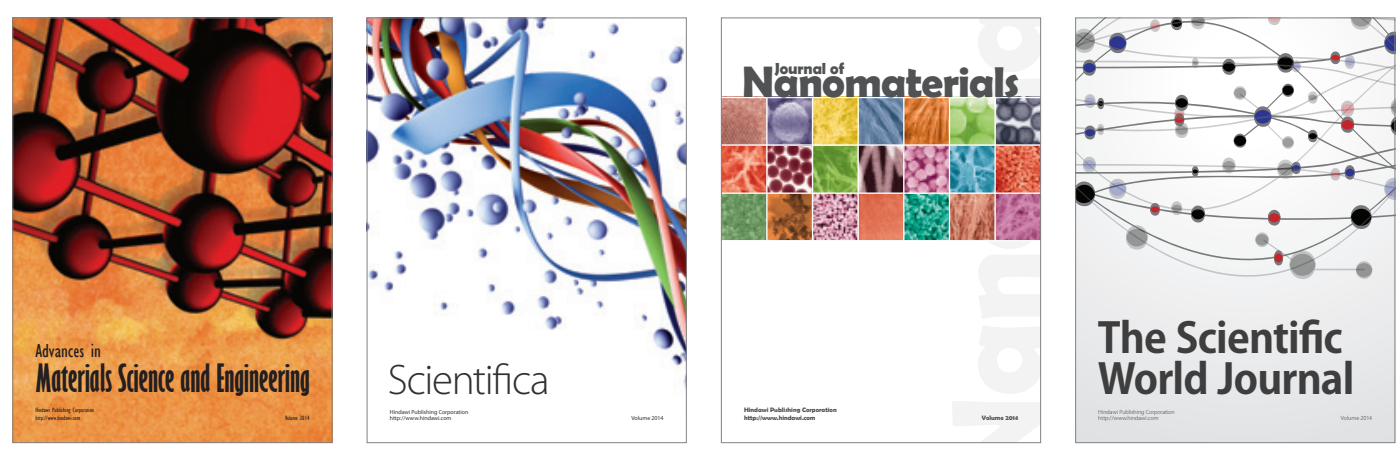

\section{The Scientific World Journal}
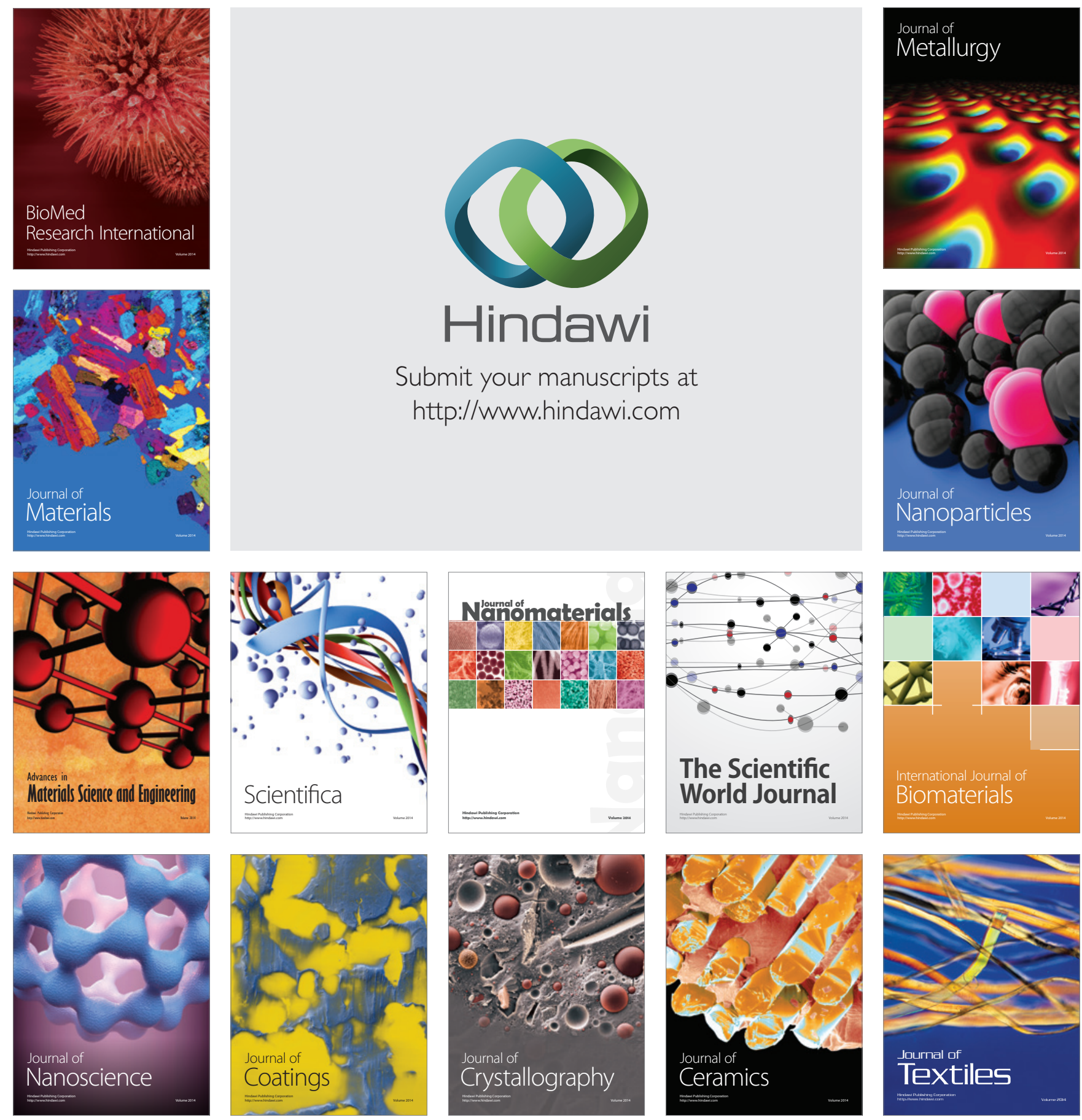